\title{
INVESTIGATION INTO THE EFFECTS OF THE GEOMETRY IN CONE DRIVEN MIDRANGE HORNS \& PHASE PLUGS
}

\author{
LT MacDonald University of Salford, Manchester, M5 4WT \\ JA Hargreaves University of Salford, Manchester, M5 4WT
}

\section{INTRODUCTION}

In professional audio, compression drivers and horns are almost always used for high frequency sound reproduction to increase sensitivity and control directivity. Horns are essentially acoustic transformers, and work by helping to match the impedance between driver and the air, leading to increased efficiency. This increase can be quite dramatic; $0.5 \%$ to $5 \%$ is common for direct radiators, whereas a well-designed compression driver may reach $20 \%$ to $40 \%$ efficiency ${ }^{1}$.

The same principal can be applied to achieve efficiency gains with paper cone drivers. But while traditional lumped parameter theory models horns accurately at low frequencies, in the mid to high frequency region the behaviour becomes more complex and the theory is much less accurate. Consequently, many of complexities of the design after the horn expansion are still considered by many to be a 'Black Art'. This is especially the case in midrange horns where phase plugs are used which vary tremendously in size and shape, sometimes up the length of the horn. These phase plugs could potentially be used to prevent beaming and also to control directivity, since they allow the expansion of the horn to be maintained whilst controlling the flare of the horn walls.

There is relatively little academic literature available on using large phase plugs in midrange horns. This is likely due to the innovation being quite recent, compared at least to direct radiating cones and compression drivers, and to most research output being wrapped up in commercial products. Geometric constraints also mean that the horn and the phase plug merge into one large design problem, as opposed to traditionally compression drivers, and their integral phase plugs, which are usually designed independently to the horns. An efficient pipeline for constrained parameterised horn design therefore has potential benefits in streamlining loudspeaker product design.

Usually a midrange horn will be part of a multiple driver system and is only required to cover a limited band of frequencies. Although the required frequency range varies from system to system, they typically sit between a mid-bass or bass section and a high frequency compression driver. Operating bandwidth can therefore be from around $150 \mathrm{~Hz}-500 \mathrm{~Hz}$ to around $1.5 \mathrm{kHz}-6 \mathrm{kHz}$. This begins at least an octave below most compression drivers yet can extend well into the critical vocal frequency range. When combined with practical constraints on cabinet size and the geometry of the drivers, this means midrange horns present a unique design challenge.

Despite these differences, midrange horns that radiate into a throat smaller than the diaphragm can also be classed as a type of compression driver ${ }^{2}$. This similarity means that design concepts and analysis developed for traditional compression drivers may also give benefits here. For example, there has recently been increased interest in considering how eigenmodes within a compression driver chamber and phase plug affect performance ${ }^{3}$. This can occur in midrange horns too, though is less of an issue than it is for high frequency compression drivers because the wavelengths in the required operating bandwidth are longer. Cone breakup also limits the benefits that may be achieved through more complicated phase plug designs intended to operate to higher frequencies. Consequentially, midrange horns commonly only have a single radial exit around a central phase plug with no other channels through it. This type of horn design is investigated in this paper.

Reference 3 also demonstrated the benefits of topology optimisation through numerical simulation for compression drivers and tweeters. "Topology optimisation" means that the geometry is parameterised, i.e. defined based on a finite set of parameters, such that adjusting these causes it to be deformed in a continuous and predictable way. The values of the parameters can then be optimised, yielding a geometry that is optimal within the space defined by the starting geometry and range of possible deformations. 
This paper applies a similar approach to investigate the aspects of cone driven midrange horn and phase plug geometry, though ranges of deformation will be studied to understand their effect instead of used in an automatic optimisation algorithm. The numerical methods fully model wave propagation in the horn, which is not fully captured by traditional lumped parameter models. The numerical acoustic model is also coupled to a lumped parameter model of the loudspeaker's electromechanical behaviour, such that radiation loading is also taken fully into account.

Section 2 will outline the methodology adopted, including the numerical method and loudspeaker selected, geometry parametrisation and constraints placed, and metrics used to quantify performance. Section 3 will show and analyse the results for a series of connected studies. Section 4 will draw conclusions on the success of the methodology and make suggestions for future work.

\section{METHODOLOGY}

The chosen methodology is a form of virtual prototyping and comprises:

i. Parameterisation of the geometry;

ii. Simulation using numerical methods with a range of topological deformations;

iii. Evaluation of parameterised designs through metrics and examination of detailed results.

The horn was designed for a real loudspeaker that is described in section 2.1. The choice of simulation algorithm is given in section 2.2, starting geometry and deformation parameters in section 2.3 and metrics in section 2.4. It was originally planned to include measurement of a 3D printed prototype horn in the research, but this was not possible due to COVID-19.

\section{$2.1 \quad$ Loudspeaker Driver}

A 6.5-inch B\&C 6NSM51 driver ${ }^{4}$ was chosen for the studies. It is a sensitive driver with a powerful motor and low $Q_{T S}$ meaning it's especially suitable for midrange horn loading. Notably it features an integral rear chamber and inverted dust cap, both of which simplify mounting design (see Figure 2).

Since the Thiele-Small parameters of drivers can vary from specifications given by the manufacture, the driver procured was measured using the Klippel Analyzer 3 Linear Parameter Measurement ${ }^{5}$ system at Tymphany's UK Design Center. Note that these measurements include chamber compliance and damping within $C_{M D}$ and $R_{M D}$, hence it is not necessary to model these separately.

Direct radiation into a half-space, with the driver flush-mounted in a planar baffle, was also measured. Simulation of the same configuration in COMSOL Multiphysics allowed the numerical model of the driver to be validated. Sensitivity as SPL at $1 \mathrm{~m}$ for $1 \mathrm{~W}$ agreed within $2 \mathrm{~dB}$ up to $3 \mathrm{kHz}$. Above this, the measured results deviated from simulation. Further measurements taken with a Polytec PSV-400 laser vibrometer system at the University of Salford suggest that this is due to the presence of non-pistonic cone breakup modes (see right of Figure 2), which aren't included in the lumped parameter model of the driver. This is therefore an upper limit of the frequency range in which our simulations are valid. However, simulations above this frequency are still of interest because they show the performance of the horn if used with a driver with a higher break up onset.
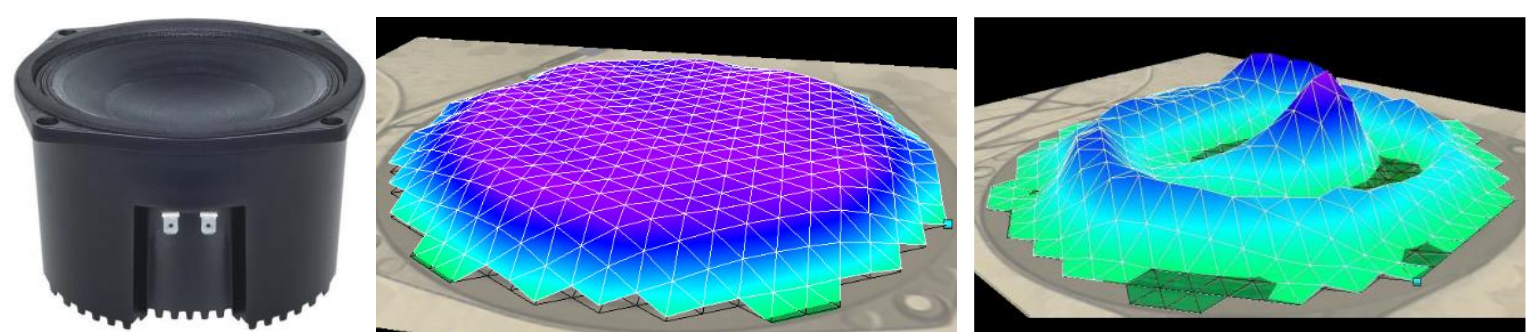

Figure 1. Left: photo of B\&C 6NSM51 driver (from ref. 4). Middle and right: Plots of cone velocity respectively showing pistonic behaviour at $300 \mathrm{~Hz}$ and cone breakup at $4 \mathrm{kHz}$.

Vol. 42. Pt. 3. 2020 

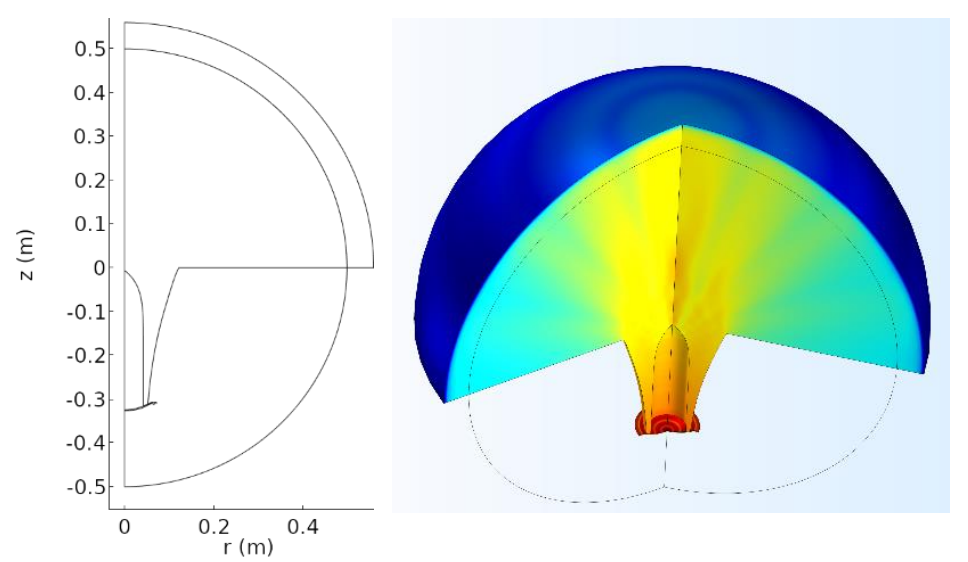

Figure 2: 2D geometry slice and SPL response in the revolved axisymmetric geometry

\section{$2.2 \quad$ Numerical Modelling Algorithm}

Simulation of the acoustic behaviour was performed using the Finite Element Method (FEM). This is a well-established simulation method within Engineering, and has been widely used in electroacoustics, including for 'multiphysics' models of loudspeakers ${ }^{6}$. Here however, a lumped parameter model ${ }^{7}$ of the driver was preferred, due to the ease of including measured Thiele-Small parameters. This was coupled to the FEM model at the cone through a velocity boundary condition and a spatial integral of pressure, which gives force acting back on the driver from the media. The horn was modelled as embedded in an infinite rigid baffle and a Perfectly Matched Layer (PML) was used to truncate the simulation domain anechoically. Simulations were performed in the frequency domain, with equilibrium air density and speed of sound $1.21 \mathrm{~kg} / \mathrm{m}^{3}$ and $343 \mathrm{~m} / \mathrm{s}$ respectively. The mesh used elements that were smaller than $\lambda / 5$ at the highest frequency. COMSOL Multiphysics ${ }^{8}$ was the software chosen to perform these simulations and undertake postprocessing.

\subsection{Parameterisation of geometry and constraints on geometries considered}

Topology optimisation using numerical methods has great potential, but case must be taken to reduce the number of parameters being optimised over else it can fast become unwieldy. It is therefore essential to place constraints on the scope of the study to limit the number of parameters and make it tractable. Here the objective is to study the effect of several features of the geometry, so the others must be fixed at sensible and realistic values. These choices, and associated simplifying assumptions, are as follows.

\subsubsection{Axisymmetric geometry and simulation}

The geometry will be assumed to be axisymmetric i.e. a cross-section rotated around an axis (see Figure 2 overleaf). This is a substantial limitation, given that most commercial horns are designed to have a rectangular aperture, but is one that yields substantial simplifications to geometry definition and vastly reduces the cost of numerical simulation. Loudspeakers are axisymmetric in nature, and horns can be too, so this takes advantage of that. Note that, because driver break-up is not being considered, only the zeroth-order azimuthal mode number was modelled.

\subsubsection{Geometry parameterisation of the horn and phase-plug}

A substantial body of literature exists reporting the effects of different horn expansions and comparing those is not the aim here. Therefore, a simple exponential expansion was used for all the horn simulations, whilst changes to other parts of the geometry were investigated. Horn area $S(z)$ versus axial coordinate $z$ is therefore defined:

$$
S(z)=S_{\mathrm{M}} \mathrm{e}^{m z}
$$

where $S_{\mathrm{M}}$ is the mouth area of the horn and $m$ is the exponential horn flare constant. This can be found by $m=\ln \left(S_{\mathrm{M}} / S_{\mathrm{T}}\right) / L$, where $S_{\mathrm{T}}$ is the throat area of the horn and $L$ is the length of the horn. Note that $z$ is zero on the front baffle and negative in the horn, so Eq. 1 horns for $-L \leq z \leq 0$.

Vol. 42. Pt. 3. 2020 
Following most commercially available cone-driven midrange horns, this research considers horns with either no phase plug or with a single annular exit phase plug. In the absence of a phase plug, the radius of the outer horn wall $r_{\mathrm{o}}$ at varying $z$ could be simply found by $r_{\mathrm{o}}(z)=\sqrt{S(z) / \pi}$. But when a phase plug is present, the area it occupies must be factored into the equation for $r_{0}$, such that the correct horn area profile is maintained. Outer horn wall radius $r_{\mathrm{o}}$ is therefore defined:

$$
r_{\mathrm{o}}(z)=\sqrt{r_{\mathrm{P}}^{2}(z)+S(\mathrm{z}) / \pi},
$$

where $r_{\mathrm{P}}(z)$ is the radial profile chosen for the phase plug. This equation will automatically deform the outer horn wall to compensate for the shape of the phase plug, as can be seen in later figures.

To allow a range of reasonable phase plug shapes to be controlled from a single parameter, the radial profile of the phase plug $r_{\mathrm{P}}$ was chosen to follow a power law:

$$
r_{\mathrm{P}}(z)=r_{\mathrm{PT}} \times\left(1-\left(\frac{z+L}{L_{\mathrm{P}}}\right)^{\alpha}\right)
$$

Here $L_{\mathrm{P}}$ is the length of the phase plug and $r_{\mathrm{PT}}$ is its radius at the horn throat where it meets the loudspeaker. $\alpha$ is a factor that controls the curvature of the phase plug. When $\alpha=1$ it will be conical, whereas $\alpha>1$ means that it will curve outwards, becoming bulbous, or taper inwards, becoming pointy. The effect of this parameter is illustrated in the figures in section 3.3.

A horn length $L$ of $310 \mathrm{~mm}$ and mouth area of $0.046 \mathrm{~m}^{2}$ was chosen as being realistic for a 6.5 " midrange horn in a multi driver system. The horn throat area $S_{\mathrm{T}}$ was set to $0.003 \mathrm{~m}^{2}$, giving a compression ratio of 4.5. The resulting exponential expansion rate has a theoretical cut off frequency of $240 \mathrm{~Hz}$, but in practice the this is around $300 \mathrm{~Hz}-350 \mathrm{~Hz}$ when the horn is truncated to length $L$. This coincides with the chosen driver's natural low frequency roll off. Although a long and narrow horn with a truncated expansion, as simulated here, can cause slight ripples in the frequency response, it is used in many commercial PA midrange horns because it allows efficient horn loading down to a lower frequencies without the horn becoming too large.

An initial study was undertaken to explore the effect of the curvature of the back of the phase plug. It was found that the flattest frequency response was achieved by having the plug follow the contour of the cone with a fixed offset, as is done in traditional compression driver design. This was retained for all studies shown herein, and the distance between the cone and the phase plug was $2.4 \mathrm{~mm}$.

This is surmised in Table 1 below. Parameters that remained to be varied and investigated were:

1. The radius of the phase plug at its base $r_{\mathrm{PT}}$;

2. The length of the phase plug $L_{\mathrm{P}}$;

3. The shape of the phase plug via its curvature coefficient $\alpha$.

\begin{tabular}{|c|c|c|c|c|c|}
\hline Parameter & Value & Description & Parameter & Value & Description \\
\hline$r_{\mathrm{PT}}$ & varied & $\begin{array}{l}\text { Radius of the phase } \\
\text { plug at throat }\end{array}$ & $S_{\mathrm{T}}$ & $0.003 \mathrm{~m}^{2}$ & Horn throat area \\
\hline$L_{\mathrm{P}}$ & varied & Length of phase plug & $S_{\mathrm{M}}$ & $0.046 \mathrm{~m}^{2}$ & Horn mouth area \\
\hline$\alpha$ & varied & $\begin{array}{l}\text { Phase plug curvature } \\
\text { coefficient }\end{array}$ & $S_{\mathrm{D}}$ & $0.014 \mathrm{~m}^{2}$ & $\begin{array}{l}\text { Driver diaphragm } \\
\text { area }\end{array}$ \\
\hline$L$ & $310 \mathrm{~mm}$ & Length of Horn & $L_{P G}$ & $2.4 \mathrm{~mm}$ & $\begin{array}{l}\text { Gap between phase } \\
\text { plug and driver }\end{array}$ \\
\hline
\end{tabular}

Table 1: Summary of Horn Geometry Parameters

Vol. 42. Pt. 3. 2020 


\subsection{Performance Metrics}

A set of appropriate performance metric aids interpretation of results and is essential if undertaking full numerical optimisation. To characterise horn performance the following metrics where used:

\subsubsection{Average sound intensity level inside intended dispersion angle range}

The power radiated by the horn with a $1 \mathrm{~W}$ power input to the driver was characterised by taking an average of sound intensity over an intended dispersion angle range. This was taken to be $60^{\circ}, 30^{\circ}$ either side of the axis of symmetry, a typical value for directional midrange horns. It is better to look at power radiated over a range of angles, compared e.g. to just on axis, since this gives a broader picture and is less susceptible to variations at some specific angle. Averaging is performed in Intensity units and then is converted to Sound Intensity Level (SIL) for plotting.

\subsubsection{Bandwidth}

The bandwidth or usable frequency range is an important characteristic of a midrange horn driver coupled system because it depicts how the horn will be integrated into the rest of the system.

$f_{\mathrm{L}}$ and $f_{\mathrm{H}}$ are the low and high cut-off frequencies of the system. They are computed on the average sound intensity level inside the dispersion angle (above). $f_{\mathrm{L}}$ and $f_{\mathrm{H}}$ are defined as the first frequencies in which the response drops to $-3 \mathrm{~dB}$ from average pass band level. Because the horn length and expansion were kept the same, it was unlikely that $f_{\mathrm{L}}$ will change significantly.

\subsubsection{Directivity}

The beamwidth is used to characterise the directivity of the horn. It was defined as the narrowest angle at which the SPL response reaches $-6 \mathrm{~dB}$ from its on-axis response.

\section{$3 \quad$ RESULT AND ANALYSIS}

This section reports the results of the three connected studies, which each investigate the effect of one of the three identified geometry parameters while keeping the other two fixed.

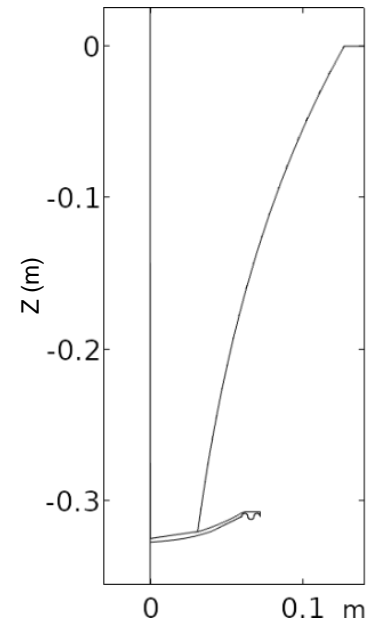

a) $r_{\mathrm{PT}}=0 \mathrm{~mm}$

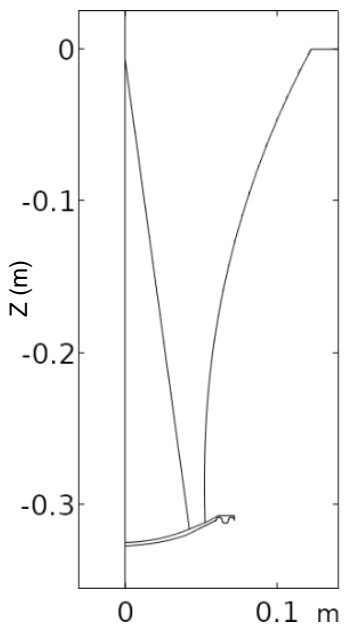

b) $r_{\mathrm{PT}}=42.5 \mathrm{~mm}$

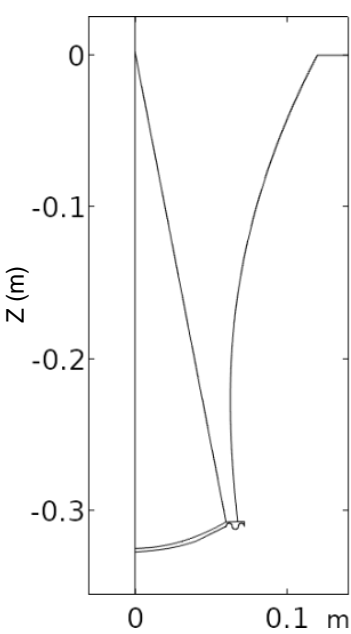

c) $r_{\mathrm{PT}}=60 \mathrm{~mm}$

Figure 3: Cross section of the horn geometry showing three different values of $r_{\mathrm{PT}}$

Vol. 42. Pt. 3. 2020 


\subsection{Effect of the Phase Plug Throat Radius $r_{\mathrm{PT}}$}

In this first study, the effect of the radius of the base of the phase plug from zero - meaning there was no phase plug at all - up to a radius of $60 \mathrm{~mm}$ was investigated. The other two parameters were chosen as $\alpha=1$ and $L_{\mathrm{P}}=L$, meaning the phase plug was conical and the same length as the horn. The geometry this creates is shown in Figure 3. Increasing $r_{\mathrm{PT}}$ moves the position of the annular channel exiting the compression chamber outwards. The throat area was kept constant, matching Table 1, so the channel narrows as it moves outwards, compensating for increasing circumference.

Simulations were performed with a sweep of $r_{\mathrm{PT}}$ values. The average intensity level within the $60^{\circ}$ dispersion angle is shown in Figure $4 \mathrm{a}$ for five selected values of $r_{\mathrm{PT}} . f_{\mathrm{H}}$, the $-3 \mathrm{~dB}$ high frequency cut-off point of this, is shown in Figure $4 \mathrm{~b}$ for a finer sweep of $r_{\mathrm{PT}}$ values.

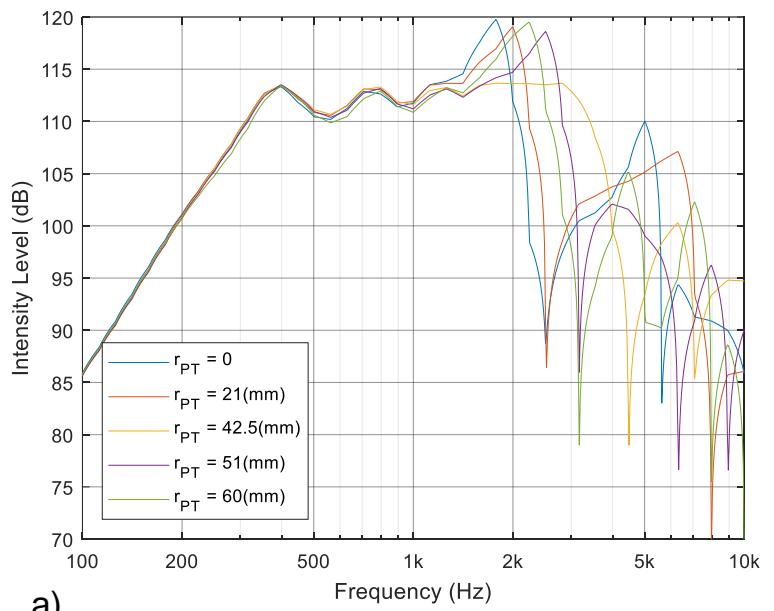

a)

Figure 4: Effect of varying $r_{\mathrm{PT}}$ : a) SIL,

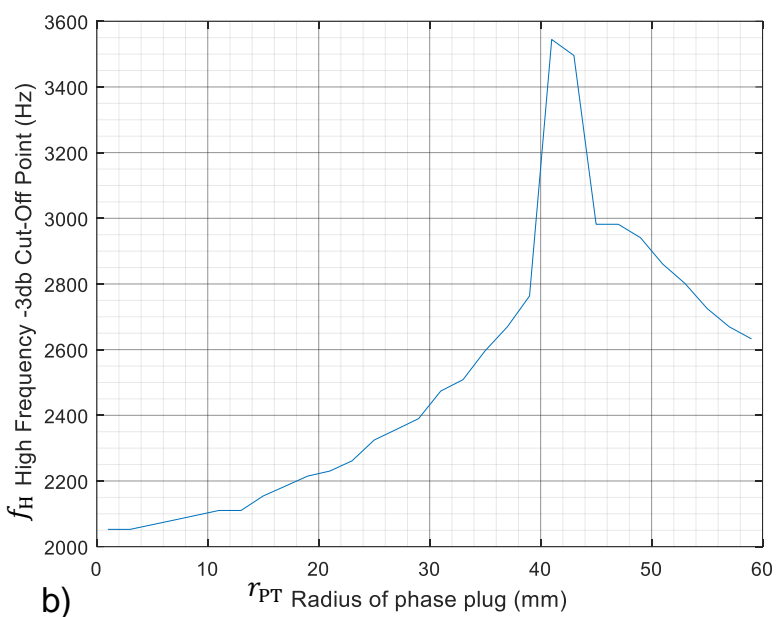

b)

Figure 4a shows that $r_{\mathrm{PT}}$ starts to have a significant effect around $1 \mathrm{kHz}$. Above this, most SIL trends can be seen to rise by around $8 \mathrm{~dB}$ and then dip suddenly to a sharp null. The exception to this is $r_{\mathrm{PT}}=42.5 \mathrm{~mm}$, which remains much flatter and then dips at a higher frequency around $3.5 \mathrm{kHz}$. Figure $4 \mathrm{~b}$ shows the values of $f_{\mathrm{H}}$ for these and other results, and confirms through its finer sweep that the maximum value of $f_{\mathrm{H}}$ occurs when $r_{\mathrm{PT}}$ is $42.5 \mathrm{~mm}$.

Exit channel position can have such a big effect on the high frequency response of a horn because it interacts with modes in the compression cavity, as was studied for traditional compression drivers in ref. 3. To test this hypothesis, an eigenfrequency study was performed on a sealed version of the compression cavity. The SPL distribution of the first order radial mode is shown Figure 4.

Overlaid on the SPL colour scale in Figure 4 are two thin black lines. These show the inner and outer radii of the channel when $r_{\mathrm{PT}}=42.5 \mathrm{~mm}$. It is evident that the pressure minimum of this mode is aligned with the exit channel of the cavity with this choice of $r_{\mathrm{PT}}$. This highlights the importance of ref. 3 when choosing the position for a compression cavity exits in relation to cavity modes.

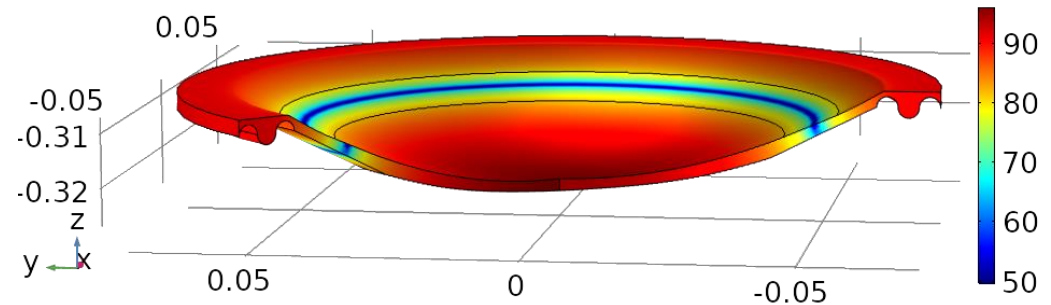

Figure 5: 3D plot of the SPL distribution (see colour bar) of the first eigenmode (2695 Hz)

Vol. 42. Pt. 3. 2020 
Furthermore, with use of eq. 2, the centre of the channel can be shown to have a radius of $47.6 \mathrm{~mm}$. The arc length of the cavity was computed to find what radius it would have if flattened out; this is $75.3 \mathrm{~mm}$. Dividing these gives a ratio of exit centre radius to effective cavity radius of 0.632 . Locating the channel at this radius should minimise colouration of the horn output by the response of this cavity mode, hence $r_{\mathrm{PT}}=42.5 \mathrm{~mm}$ is retained for subsequent studies. This placement is consistent with the channel design methodology of Smith ${ }^{9}$ when a single channel is used. Figure $4 a$ shows that other placements give peaks and sharp nulls in the averaged frequency response.

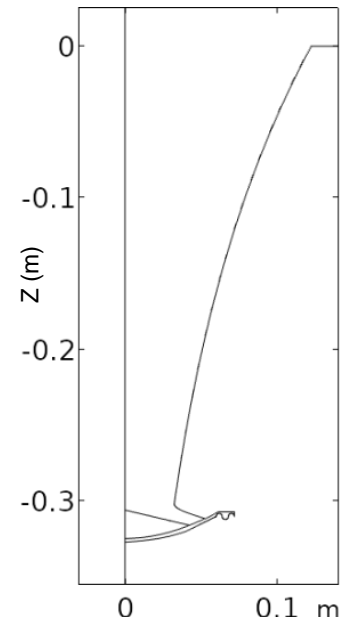

a) $L_{\mathrm{P}}=10 \mathrm{~mm}$

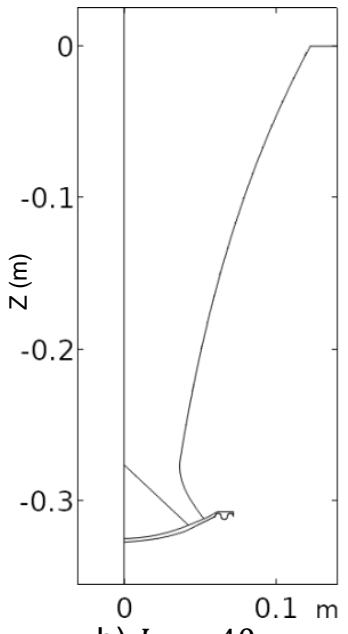

b) $L_{\mathrm{P}}=40 \mathrm{~mm}$

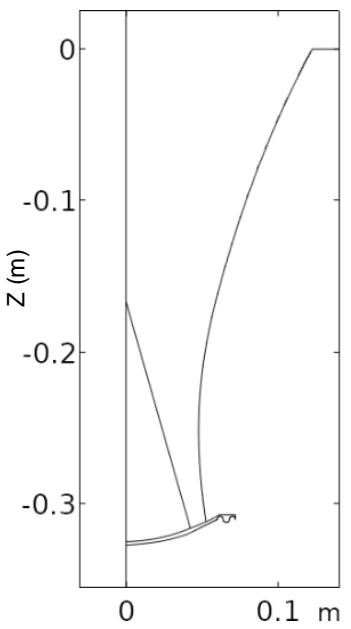

c) $L_{\mathrm{P}}=160 \mathrm{~mm}$

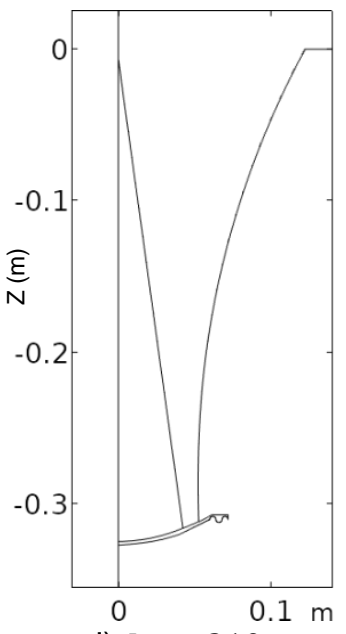

d) $L_{\mathrm{P}}=310 \mathrm{~mm}$

Figure 6: Cross section of the horn geometry showing three different values of $L_{\mathrm{P}}$

\subsection{Effect of the Phase Plug Length $L_{\mathrm{P}}$}

The effect of phase plug length $L_{\mathrm{P}}$ will now be studied. This can vary from lengths much smaller than $L$, which can give quite flat plug, to $L_{\mathrm{P}}=L$, which means the plug will extend all the way to the front of the horn, as was done in the previous study. $\alpha=1$ is retained so the horn is conical. Figure 6 shows examples of the effect of $L_{\mathrm{P}}$ on the cross-sectional geometry of the horn. It can also be seen that small values lead to an abrupt change in the direction of the channel to where the plug ends, whereas longer plugs combine with the exponential flare to give a smooth transition.

Figure 7 a shows the average intensity level within the $60^{\circ}$ dispersion angle for the four selected values of $L_{\mathrm{P}}$ shown in Figure 6. A finer sweep of values was performed, but these are chosen to present since they are indicative of the main trends observed. Figure $7 \mathrm{~b}$ shows the beamwidth.
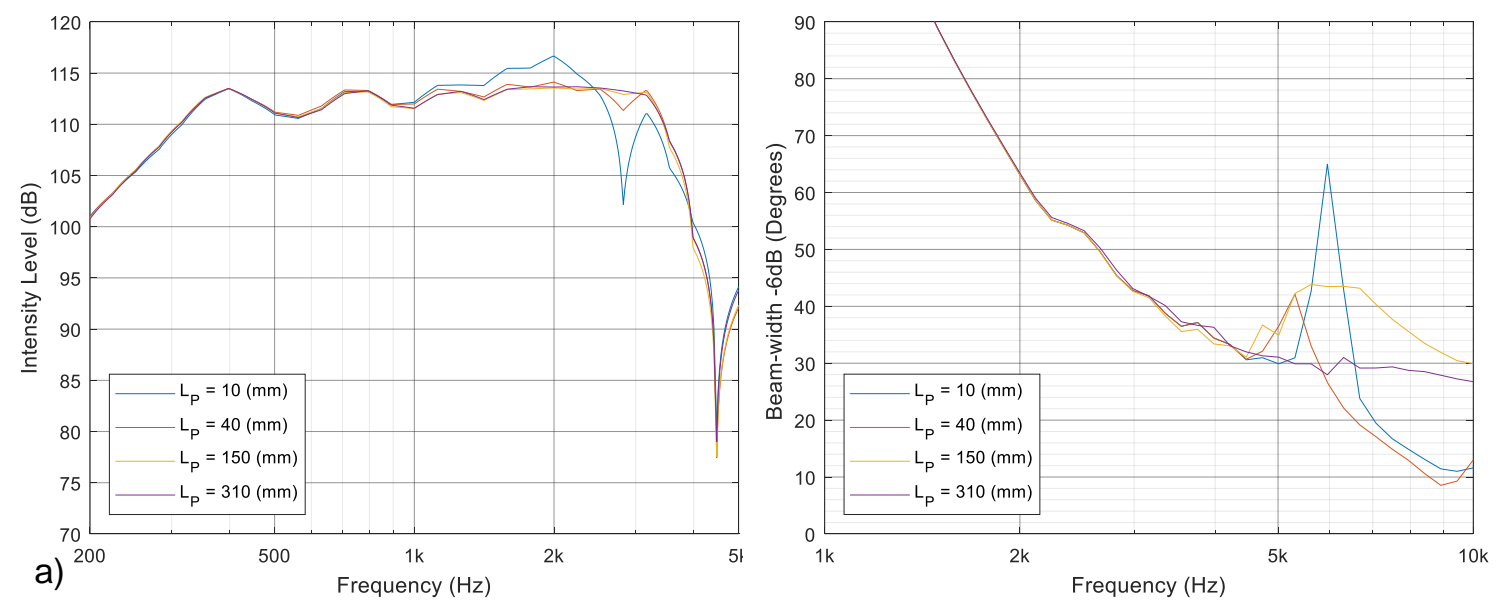

Figure 7: Effect of varying the length of phase plug $L_{P}:$ a) SIL, b) Beamwidth

Vol. 42. Pt. 3. 2020 
Figure 7a shows that $L_{\mathrm{P}}$ has a significant effect on the bandwidth of the horn for small values $(10 \mathrm{~mm}$ and $40 \mathrm{~mm})$ that give a sharp bend in the horn path. These cause deviations in the passband, which are quite exaggerated for $L_{\mathrm{P}}=10 \mathrm{~mm}$ but also present for $L_{\mathrm{P}}=40 \mathrm{~mm}$. Above $40 \mathrm{~mm}$ these rapidly vanish, and the results for $150 \mathrm{~mm}$ and $310 \mathrm{~mm}$ are nearly identical.

Figure $7 \mathrm{~b}$ shows that all four designs presented have very similar beamwidth until $4 \mathrm{kHz}$, at which point they diverge with a narrowing in directivity seen for the shorter phase plugs. The $150 \mathrm{~mm}$ design shows an interesting broadening in directivity, but the choice of $L_{\mathrm{P}}$ with the most uniform directivity at high frequencies is $310 \mathrm{~mm}$, an equal length to the horn. Note that these differences are above the intended operating bandwidth with this driver, but are still interesting since the FEM model remains valid here and the results could be applicable to a driver with a higher roll-off.

In summary, it has been seen that using too short a phase plug in this design paradigm causes an abrupt curve in the geometry of the horn wall where the phase plug ends. This causes undesirable artefacts in the frequency response, so is best avoided, meaning a longer phase plus is preferred. Both the longer designs, $L_{\mathrm{P}}=150 \mathrm{~mm}$ and $310 \mathrm{~mm}$, are therefore carried forwards for evaluation in to the third study where the effect of curvature of the phase plug will be studied.

\subsection{Effect of the curve of the phase plug}

The effect of the phase plug curvature will now be studied. This is controlled by the coefficient $\alpha$ as specified in Eq. 3. Two phase plug lengths will be studied: $L_{\mathrm{P}}=150 \mathrm{~mm}$ and $310 \mathrm{~mm}$.

\subsubsection{Curvature with a phase plug around half the horn length $L_{\mathrm{P}}=150 \mathrm{~mm}$}

The effect of the coefficient $\alpha$ on the phase plug geometry can be seen in Figure 10. $\alpha=1$ produces a phase plug that is conical, whereas $\alpha=0.7$ produces one that curves inwards and is 'pointier'. $\alpha=$ 4 and $\alpha=9$ produce outward curving ones that are more 'bulbous'. Eq. 2 adjusts the profile of the horn wall to compensate and maintain an exponential flare. Here it can be seen that a smooth transition results for $\alpha=0.7$ and $\alpha=1$, but for $\alpha=4$ and $\alpha=9$ the fast contraction at the end of the plug causes a 'kink' in the horn wall. This will be seen to be undesirable, but its presence alone is an interesting feature. Various commercial midrange horns have 'bulbous' phase plugs that terminate part way down the horn, but it is rare to see the wall of the horn adjusted to compensate like this. This means that their expansion rate will instead have a spike in it as the phase plug ends.

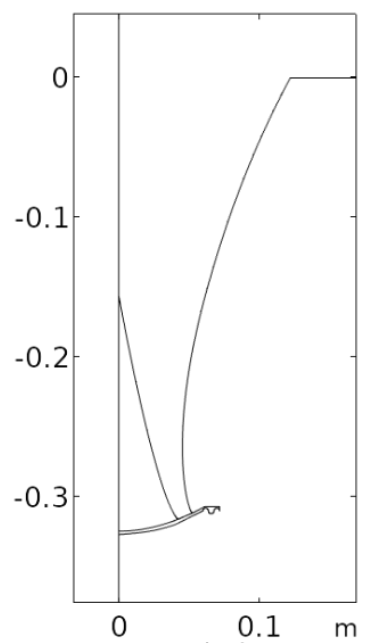

a) $\alpha=0.7$

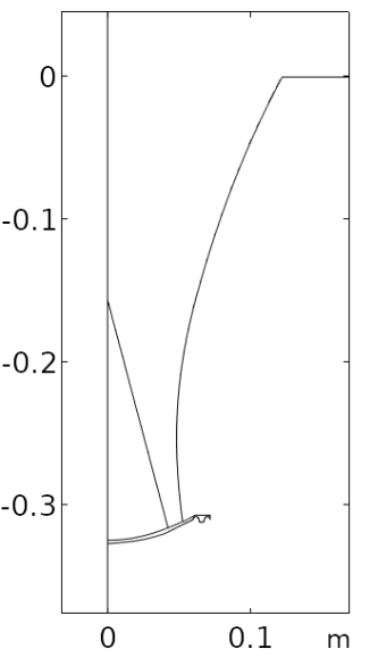

b) $\alpha=1.0$

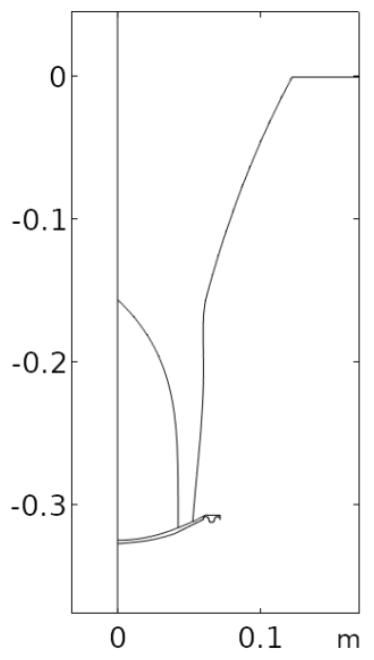

c) $\alpha=4.0$

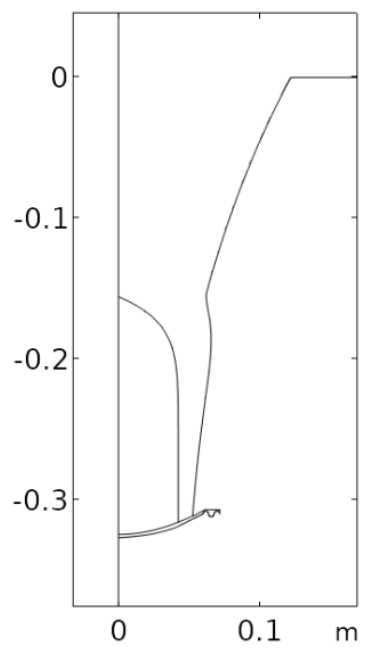

d) $\alpha=9.0$

Figure 8: Cross-section of horn geometry showing four values of $\alpha$ with $L_{\mathrm{P}}=150 \mathrm{~mm}$

Vol. 42. Pt. 3. 2020 

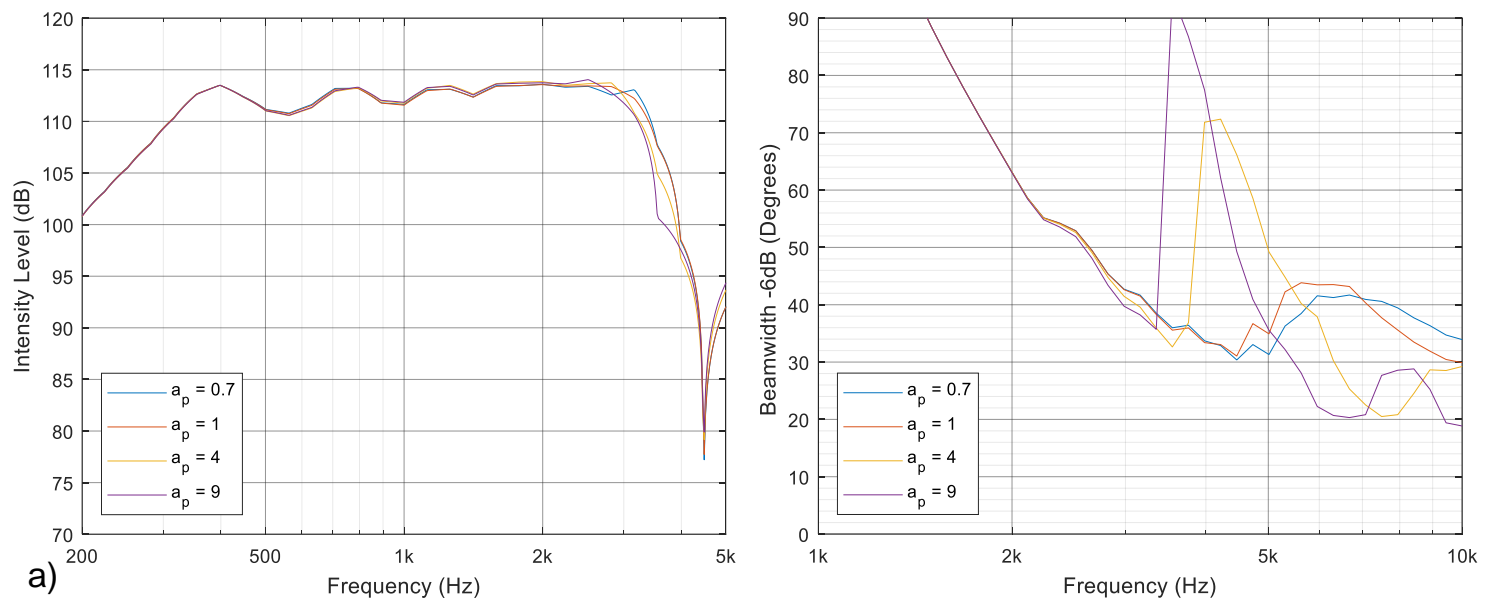

Figure 9: Effect of varying the curvature $\alpha$ when $L_{P}=150 \mathrm{~mm}$ : a) SIL, b) Beamwidth

Figure 9a shows the averaged intensity level for the four variants of the design. Changing $\alpha$ has had relatively little effect here - the only notable effect is a slight reduction in $f_{\mathrm{H}}$ for $\alpha=4$ and $\alpha=9$. But for the beamwidth results in Figure $9 \mathrm{~b}$ the difference is more marked, with both $\alpha=4$ and $\alpha=9$ showing anomalous widening of beamwidth around the cut-off frequency of the system $(3.5 \mathrm{kHz})$. The precise cause of this requires further investigation, but it seems likely that diffraction at the abrupt change in horn wall angle could play a part. The slight broadening of directivity above $5 \mathrm{kHz}$ that was seen in Figure $7 \mathrm{~b}$ for $\alpha=1$ is seen again here and occurs for $\alpha=0.7$ also, offsetting the narrowing of directivity with frequency that is expected for an exponential horn. However, as noted before, this is above the intended operating bandwidth of this system.

\subsubsection{Curvature with a phase plug running the full horn length $L_{\mathrm{P}}=310 \mathrm{~mm}$}

A set of longer phase plugs are now considered. As seen in Figure 10c \& d, extending the phase plug to the horn mouth allows the expansion rate to be maintained for the more 'bulbous' designs without introducing a 'kink' in the horn wall. This is, however, simply because that 'kink' coincides with the mouth, where an abrupt change in angle occurs already as the horn meets the baffle.

The averaged intensity level is shown in Figure 11a. Again, little different is seem between the designs, thought there is now a lift of $1-2 \mathrm{~dB}$ around $2-3 \mathrm{kHz}$ for the more bulbous designs. More difference is again seen in the beamwidth plot in Figure 11b. Here the trends for $\alpha=4$ and $\alpha=9$

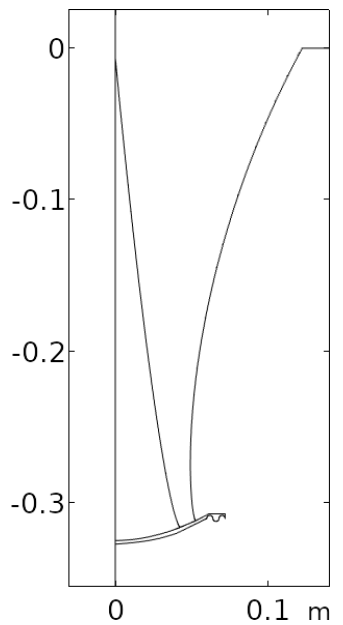

a) $\alpha=0.7$

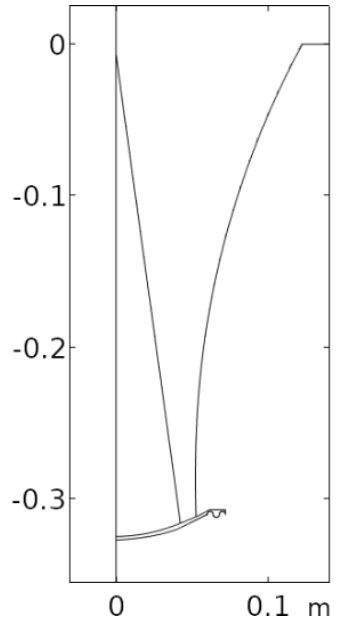

b) $\alpha=1.0$

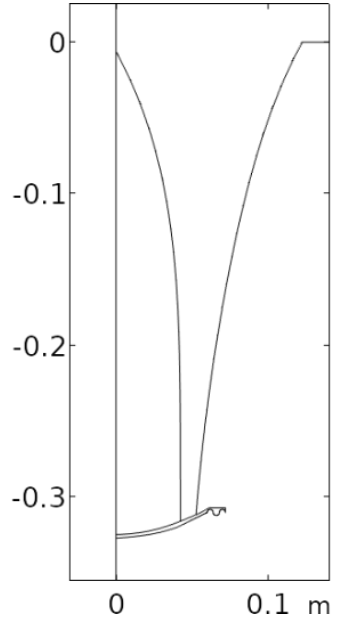

c) $\alpha=4.0$

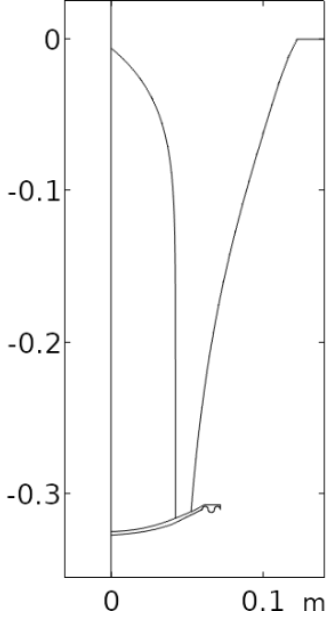

d) $\alpha=9.0$

Figure 10: Cross-section of horn geometry showing four values of $\alpha$ with $L_{\mathrm{P}}=310 \mathrm{~mm}$

Vol. 42. Pt. 3. 2020 

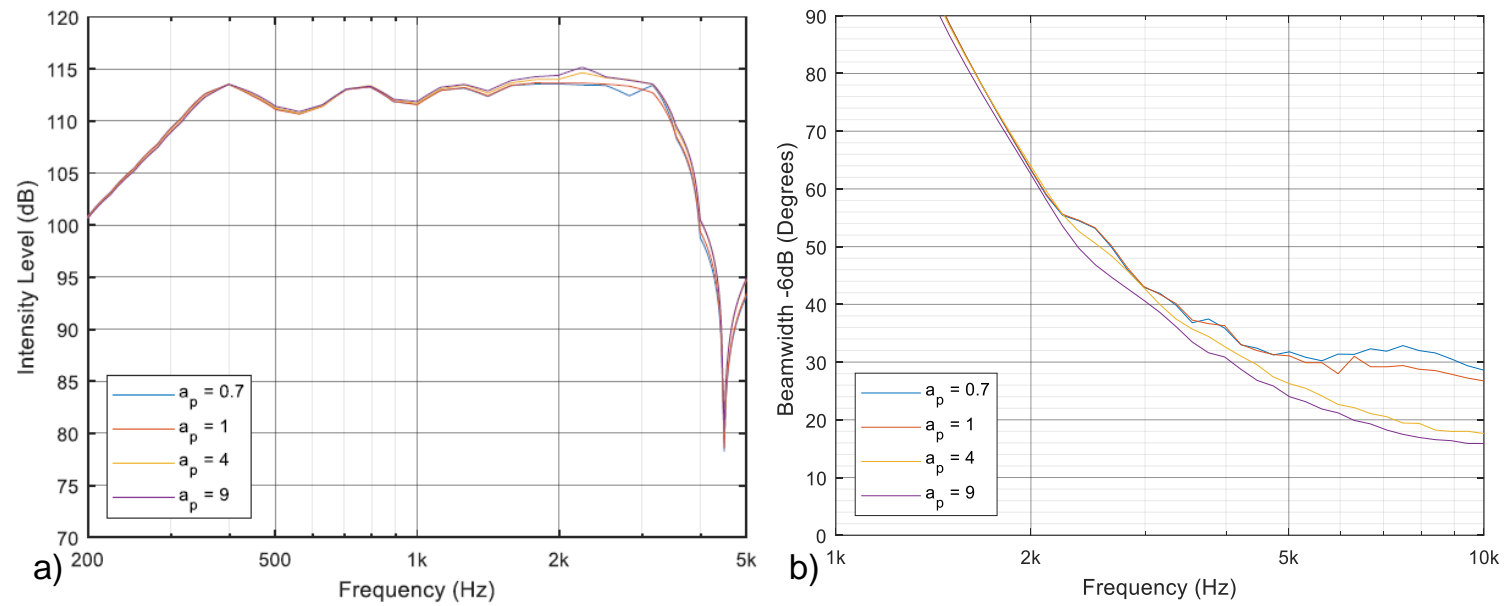

Figure 11: Effect of varying the curvature $\alpha$ when $L_{P}=310 \mathrm{~mm}$ : a) SIL, b) Beamwidth

are smooth - there are no artefacts, suggesting that those in Figure 9b were associated with the 'kink' in the horn wall. But they instead give a slight narrowing in directivity compared with the conical and inward-tapering designs arising from $\alpha=0.7$ and $\alpha=1$. Looking up to $5-10 \mathrm{kHz}$, beyond the intended operating range, the effect becomes more drastic, with the beamwidth of $\alpha=4$ and $\alpha=9$ continuing to narrow, while $\alpha=0.7$ and $\alpha=1$ achieve close to a constant $30^{\circ}$ directivity.

This is an interesting result as $\alpha=0.7$ and $\alpha=1$ more closely resemble a simplified and extended compression driver phase plug, while $\alpha=4$ and $\alpha=9$ are closer to the large phase plugs seen in many modern professional midrange horns, which are often straight or bend outwards. Notably, $\alpha=0.7$ sees the horn walls converge to a minimum radius around $5 \mathrm{~cm}$ in front of the driver.

One possible explanation for the trends in Figure 11b is that relatively large radius of the 'bulbous' phase plug near the horn mouth (Figure 10d) pushes the majority of the energy out to a larger radius, causing it to act like a ring radiator. One could also speculate that the increased curvature of the outer wall in Figure 10a is causing more sound energy to be guided round to wider angles.

To investigate this further, the directivity map (SPL normalised to on-axis, plotted against frequency and angle) are shown in Figure 12 for a) the horn with $\alpha=0.7$, and b) a more exaggerated 'bulbous' horn, where an additional outward curvature factor has been added (see inset geometry). These are compared to theoretical results for a piston in a rigid baffle ${ }^{7}$ in Figure 13. In Figure 13a, the piston is a disk with are equal to the mouth of the horn $S_{\mathrm{M}}$. In Figure 13a, this same piston has had a smaller disk $75 \%$ of the radius cut out, leaving a ring radiator with a rigid disk in its centre.

In Figure 12a it can be seen that the horn with the inwardly curved phase plug produces a single dominant lobe, with no strong sidelobes. But the horn with the 'bulbous' phase plug in Figure 12b produces a narrower main lobe and stronger sidelobes. Comparing this now to Figure 13, it can be seen that, while these figures do not match exactly those in Figure 12 (we do not expect them to), the comparison is quite revealing. The horn with the inward-tapering phase plug gives a response that is clearly closer to the disk radiator, albeit with a wider main lobe and lower sidelobes. The horn with the 'bulbous' phase plug, in contrast, more strongly resembles the radiation pattern from the disk, with a narrower main lobe and stronger sidelobes. However, these differences remain above the intended operating bandwidth of the horn, so may be unimportant in practice.

\section{DISCUSSION AND CONCLUSIONS}

Due to the complexity of horn and phase plug geometry, it can be difficult to draw definitive conclusions since there are numerous different and inter-dependent variables, even in a highly constrained space of potential designs such as this. However, some themes can be observed.

Vol. 42. Pt. 3. 2020 

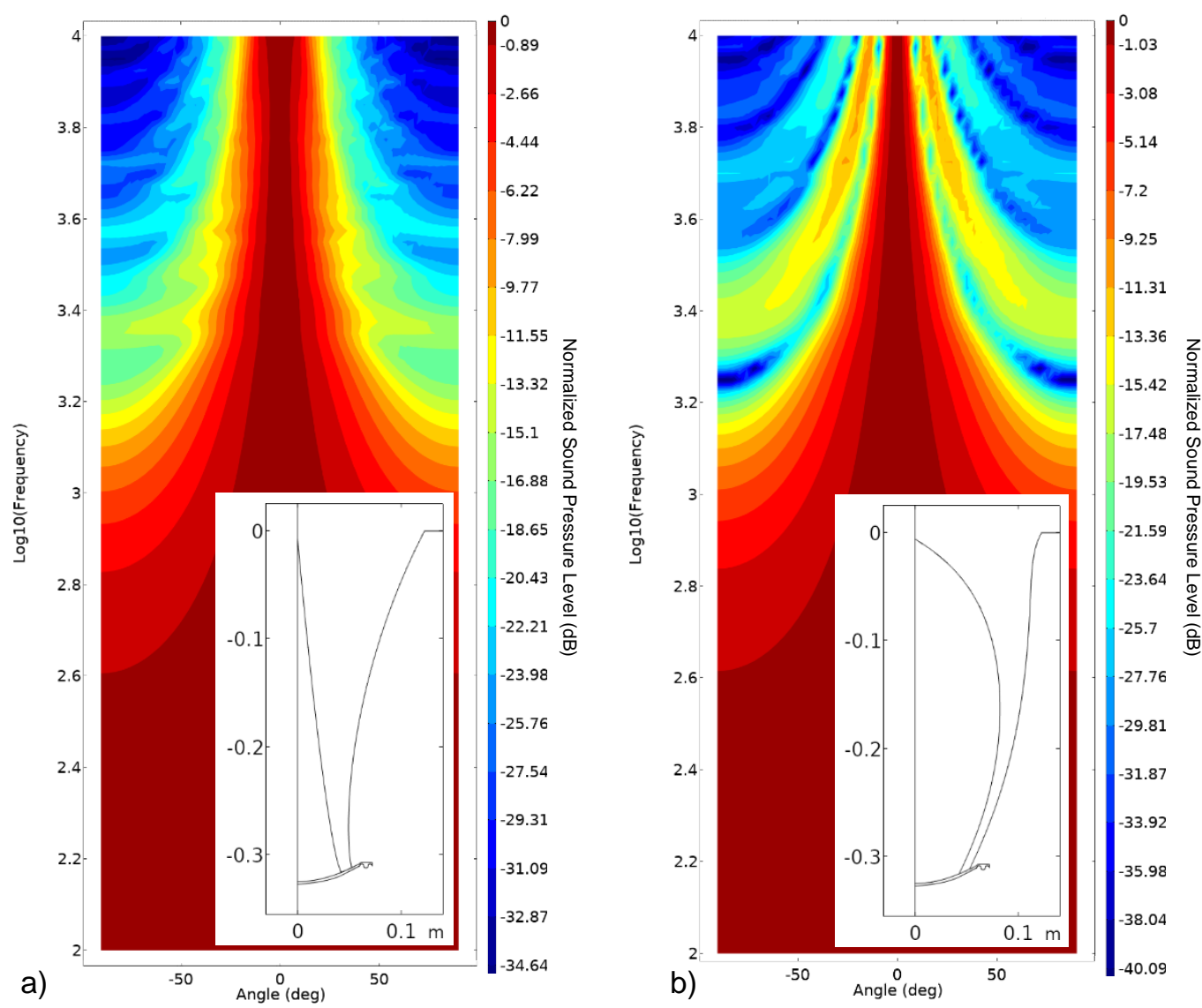

Figure 12: Geometry and directivity map comparing two very different phase plug designs
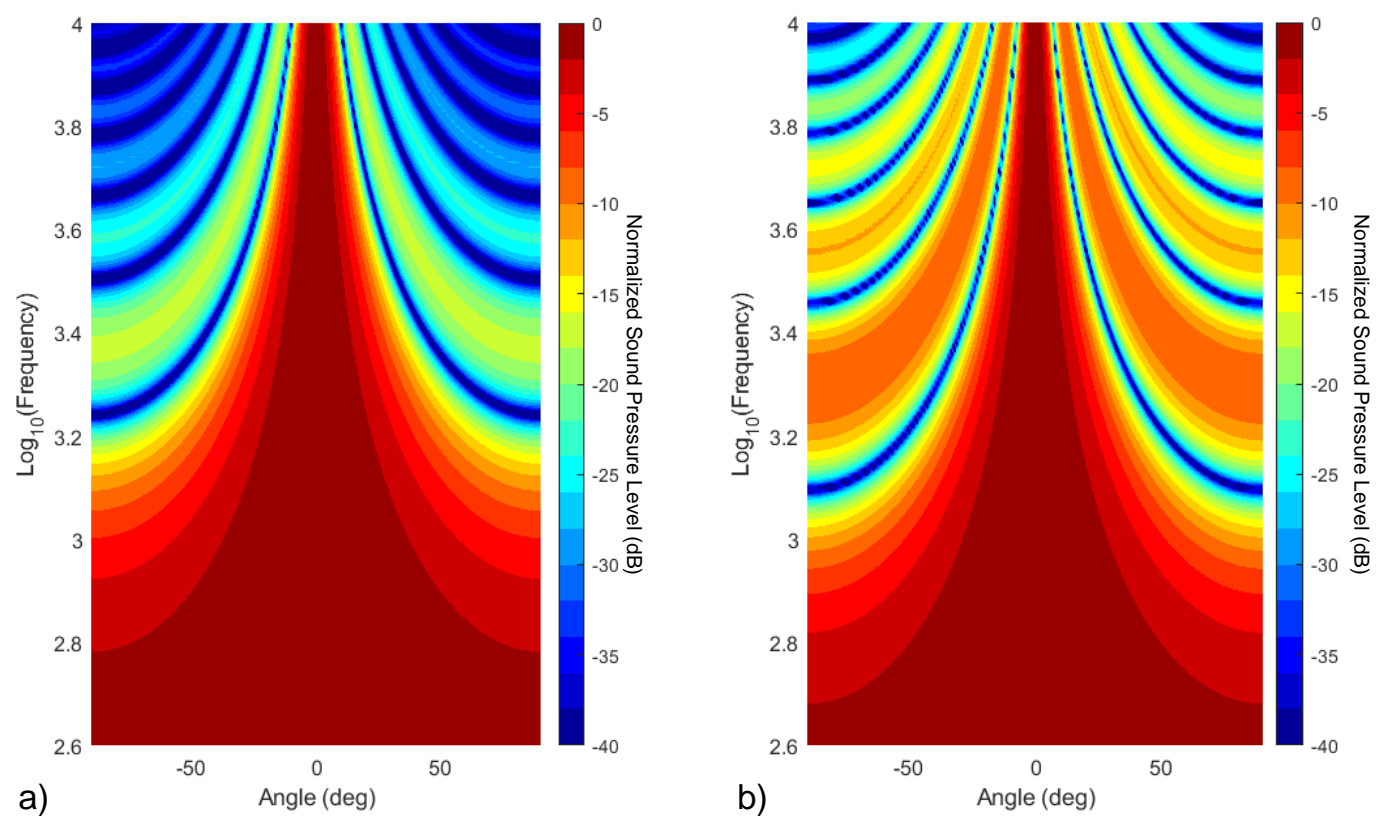

Figure 13: Directivity maps for theoretical piston radiation: a) disk, b) ring 


\subsection{The High Frequency Compression Cavity Mode Problem}

The study of radius of the phase plug at the throat showed that the placement of the annulus with respect to the cavity radius has a crucial effect on the high frequency performance of the horn. This is due to the modal response of the cavity, demonstrating that the design of a midrange horn compression cavity should be treated similarly to cavities in high frequency compression drivers.

\subsection{Design of horn and phase plug after the compression cavity}

When the horns expansion is maintained, the shape of the phase plug after the compression cavity has a relatively small effect on the intensity level within the defined dispersion angle. Unless it causes sharp bends in the horn path, the high frequency cut off point was not significantly affected.

The directivity of the coupled system was affected by the shape of the phase plug and horn wall, though not to a great degree. In most examples a change in the beamwidth was only significant above $3 \mathrm{kHz}$, though some differences were seen at lower frequencies. However, in an integrated multiway system, many midrange horns will have already been crossed over to a compression driver by $3 \mathrm{kHz}$, and/or the diaphragm in lots of midrange drivers will likely have started to break up, so this could be of limited consequence. But in the case of a larger midrange horn, the effects could be more significant. For example, if the phase plug in the horn modelled here can begin to slightly control the directivity above $3 \mathrm{kHz}$, then it would be expected that directivity control may begin at $1.5 \mathrm{kHz}$ in a horn twice the size, which could be more useful in realistic usage.

These findings lead to the conclusion that, so long as the expansion profile $S(z)$ is maintained, the design of the midrange horn and phase plug after the compression cavity has a relatively small effect on performance, and only around high frequency limit of the horn driver system does this become more significant. It also indicates that a very wide phase plug does not have a particularly detrimental effect on the performance and only slightly effects the directivity at high frequencies, so the design of them could be tailored to other needs. For example, installing a high frequency compression driver inside phase plug allows for concentric imaging from the two nested horns, and based on this work, should have a negligible effect on the performance of the midrange horn.

\section{ACKNOWLEDGEMENTS}

We are grateful to Tymphany UK for allowing us use of their Klippel Analyzer and half space anechoic chamber to profile the loudspeaker driver.

\section{REFERENCES}

1 B. Kolbrek \& T. Dunker, High-Quality Horn Loudspeaker Systems. Kolbrek Elektroakustikk, Croydon, UK. (2019)

2 H. Olsen. Elements of Acoustical Engineering. D. Van Nostrand Company, Ltd. London. (1957)

3 J. Oclee-Brown. Loudspeaker Compression-Driver Phase-Plug Design. University of Southampton, Southampton, UK. Retrieved from https://eprints.soton.ac.uk/348798/ (2012).

4 https://www.bcspeakers.com/en/products/lf-driver/6-5/8/6nsm51-8 (accessed 16/10/2020)

5 https://www.klippel.de/products/rd-system/modules/lpm-linear-parameter-measurement.html (accessed 16/10/2020)

6 R. Magalotti. A Multiphysics Approach to the Design of Loudspeaker Drivers. COMSOL Conference, Grenoble (2015)

7 L. Beranek \& T. Mellow. Acoustics Sound Fields and Transducers. Academic Press, Oxford. (2012)

8 https://www.comsol.com/ (accessed 16/10/2020)

9 B. H. Smith. An investigation of the air chamber of horn type loudspeakers. Journal of the Acoustical Society of America Vol. 25. No. 2. Pages 305-312. (1953).

Vol. 42. Pt. 3. 2020 